\title{
Calculation features of magnetic coupling in non-magnetic structures
}

\author{
Vladimir Kosulin and Irina Salova* \\ SUAI, 190000, St. Petersburg, Russia
}

\begin{abstract}
This paper presents analytical approach for computing the torque transmitted by magnetic coupling with radially polarized permanent magnets. The method for calculating magnetic couplings in nonmagnetic constructions is proposed. 2D models of magnetic couplings for finite element calculation are presented.
\end{abstract}

\section{Introduction}

Magnetic couplings are widely used in circulating pumping systems for pumping environmentally hazardous and corrosive liquids in chemical, oil and gas processing, food, pharmaceutical and other industries. In them, the magnetic field is created by permanent magnets ( $\mathrm{NdFeB}$ and $\mathrm{SmCo}$ ) installed in the leading and driven couplings. A lot of work has been devoted to the calculation of magnetic couplings, for example, [1-7], where constructions with steel magnetic cores are considered. In $[1,2]$, the influence of the permanent magnets geometry on the coupling characteristics is investigated. In [3], the influence of the pole pairs number and the air gap size on the magnitude of the torque, developed by the coupling is considered. In [6], the results of optimization of the radial magnetic couplings geometry are presented. As the optimization criterion, the volume of permanent magnets is assumed. To study and calculate coupling parameters, the authors use methods of magnetic fields mathematical modeling, which are based on solving the Laplace or Poisson equations with respect to the scalar or vector potential of the magnetic field in the investigated region.

The last time for the analysis of magnetic systems, the finite element method is widely used $[3,4,5]$. This method gives good agreement with experiment. In a number of cases, the application of these methods is difficult because of their complexity and cumbersomeness of the field theory equations. In addition, the calculation of each version of the magnetic system requires considerable time. In engineering calculations it is required to study the design of the coupling in combination with the main device. At this stage it is required to estimate the possibility of realizing a given moment for various overall dimensions of the structure as a whole. Therefore, a technique is required that makes it possible to quickly calculate the magnetic coupling maximum torque for several design variants. Such method is based on the construction and calculation of circuits for the replacement of magnetic circuits $[7,10]$.

There are designs in which the steel magnetic circuits cannot be used for operating conditions. In such designs, there is no saturation of the magnetic cores. Therefore, such constructions are most easily calculated. In such designs magnets are mounted in a holder, made of nonmagnetic material. Since the magnetic permeability of the armature approaches to the magnetic conductivity of air, the amount of useful magnetic flux is significantly influenced by scattering fluxes, which should be taken into account when calculating the magnetic flux in the air gap. The calculation of such systems is connected with the determination of the magnetic resistances of the air gaps, both between the working surfaces of the magnets and along the paths of the scattering fluxes. In this case, the problem becomes linear, since there are no sections with steel.

\section{Method content}

\subsection{Physical basics of the method}

Let us consider this method using the example of a magnetic coupling with a radial arrangement of permanent magnets. The element of the magnetic coupling system with the radial arrangement of permanent magnets is shown in Figure 1,a. Permanent magnets, magnetized radially, are shown by hatching, and the structural elements of the coupling are omitted and replaced by air gaps. In Figure 1 also indicates: $h_{m}-$ the height of a permanent magnet; $b_{1}, b_{2}$ - width of permanent magnets of outer and inner holders, respectively; $l-$ axial dimension of the coupling; $\Delta_{1}, \Delta_{1}^{\prime}, \Delta_{2}, \Delta_{2}^{\prime}$ - the distance between the sides of the magnets of the upper (1) and lower (2) holders. The expression for the torque of magnetic coupling as a function of the relative angle between the inner and outer holders has the form [8]:

* Corresponding author: salova_irine@mail.ru 


$$
M=\frac{p \pi D l \delta}{2 \mu_{0}} B_{\delta m}^{2} \sin \Theta
$$

where $D$ - the diameter of the average circle of the working non-magnetic gap $\delta$; Q-the rotating electric angle of the inner holder relative to the outer $\left(Q=p Q^{\prime}\right.$, here $Q^{\prime}$ - rotating space angle); $B_{\delta m}$ - the maximum value of induction in the middle of the air gap $\delta$ when the magnet coupling is in the idle mode $(\mathrm{Q}=0) ; p$ - the number of poles pairs; $l$ - the length of the magnet in the axial direction; $\mu_{0}=4 \pi \cdot 10^{-7} \mathrm{H} / \mathrm{m}$. This expression contains the maximum value of induction, to determine which it is required to know the induction distribution with respect to the angle $B\left(\Theta^{\prime}\right)$, which requires the solution of the field problem.

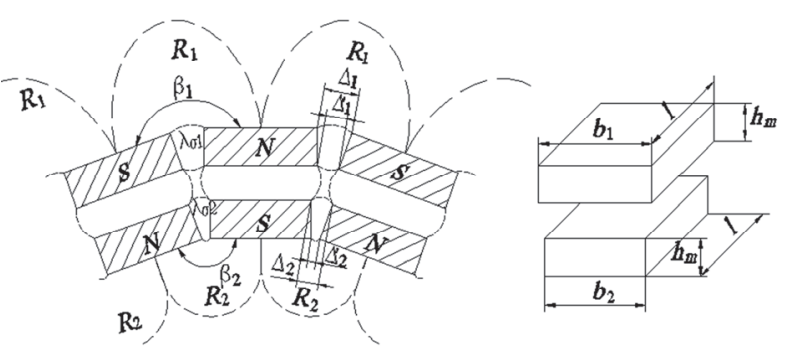

a)

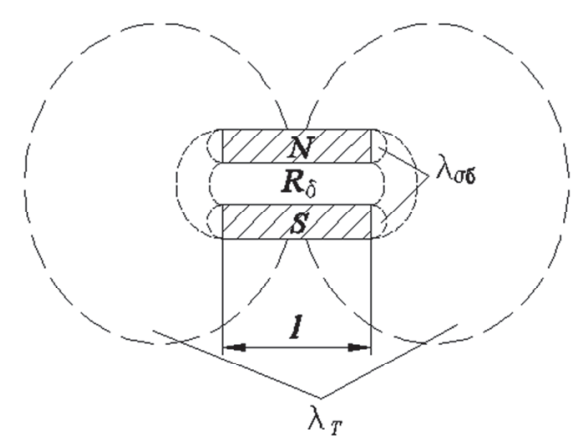

b)

Fig. 1. The distribution of magnetic fluxes of mutually perpendicular planes.

For engineering practice, this calculation meets certain difficulties because it requires the application of software packages, which is not always possible and appropriate. Let us consider the method based on the using of equivalent magnetic circuits, which will allow pre-calculation of magnetic coupling to estimate with a known degree of accuracy the magnitude of the coupling failure torque use the integral characteristics of the magnetic field - magnetic flux. To do this, in the expression for the magnetic coupling torque, we pass to the induction average value and, accordingly, to the magnetic flux $F_{\delta}$, which closes between the poles of the half couplings in the idle mode:

$$
M=\frac{2 p^{3} \Phi_{\delta}^{2} \delta}{\mu_{0} D \pi l \alpha_{t}^{2}} \sin \Theta,
$$

where $\alpha \approx b_{\text {mid }} / t$ - coefficient of pole overlap; here $b_{\text {mid }}$ - the permanent magnets arc average value of the inner and outer holders; $t$ - pole division $(t=\pi D / 2 p)$.

To calculate the magnetic flux, we construct an equivalent circuit for replacing the magnetic circuit and carry out its calculation.

On the constructive scheme of the device magnetic circuit we determine the probable path of the magnets fluxes, distinguish scattering flows and main work flow. For each flux, we calculate the magnetic conductivity (or magnetic resistance) and construct an equivalent circuit. In this case, the equivalent circuit is compiled for the main working flux, and the influence of the scattering fluxes is taken into account by the reduction factors for the magnets parameters.

In Figure 1, for the model under consideration, the dashed lines conventionally denote the boundaries of the possible paths of the corresponding magnetic fluxes in the system.

The magnetic flux of each permanent magnet consists of two components: a useful magnetic flux passing through an air gap with magnetic resistance $R_{\delta}$, and scattering fluxes along paths with magnetic conductivities $\lambda_{\sigma 1}, \lambda_{\sigma 2}$ and $\lambda_{\sigma b}$. Scattering fluxes do not participate in creating a useful flow and can be excluded from consideration by introducing the reduction coefficients $k_{\sigma}$. The calculation of the air gap magnetic conductivities for most common configurations is performed by known methods [9]. The main working magnetic flux on the outer faces of the outer and inner holders is closed along the paths of air gaps with a magnetic resistance $R_{1}, R_{2}$, and $R_{T}=1 / \lambda_{T}$ (Fig. 1 ).

Then we can assume that the useful magnetic flux of each pair of magnets, passes through the air gap, consists of two components:

- the flow in the plane perpendicular to the axis of the coupling (Fig.1,a);

- the flow in the plane passing through the coupling axis (Fig. 1,b).

The geometry of the coupling is such that the resistances $R_{1}, R_{2}$ and $R_{T}=1 / \lambda_{T}$ are comparable, this means that both magnetic flux (transverse and longitudinal) are involved in the creation of a working magnetic flux. This situation is not typical for systems with steel magnetic cores, since practically all the useful magnetic flux is closed in the transverse plane.

Consider the principle of calculating the reduction coefficient $k_{\sigma}$, which takes into account the change in the calculated magnets parameters, with the elimination of scattering fluxes.

If the equivalent scheme is presented for any magnet in the simplest magnetic system (Fig.2,a), then it will include four elements:

- actual MMF source $\left(F_{m}\right)$ - the permanent magnet;

- magnetic resistance $R_{m}$ of a permanent magnet;

- magnetic resistance $R_{\sigma}$ of scattering;

- magnetic resistance $R_{\delta}$ of the air gap for the main (useful) flow.

There will be two obvious transformations: 
- MMF source is converted into a current source and we combine the resistances $R_{m}$ and $R_{\sigma}$ into one as connected in parallel: $R^{\prime}=\frac{R_{m} R_{\sigma}}{R_{m}+R_{\sigma}}$;

- current source is converted back into the MMF source $F_{m}^{\prime}$ with internal resistance $R^{\prime}=\frac{R_{m} R_{\sigma}}{R_{m}+R_{\sigma}}$.

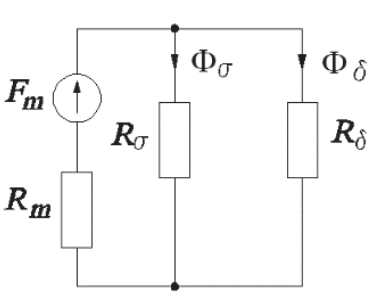

a)

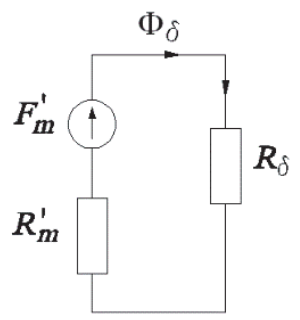

b)
Fig. 2. An equivalent magnetic circuit of the permanent magnet.

We introduce the coefficient of reduction $k_{\sigma}=1+\frac{R_{m}}{R_{\sigma}}$. Then the following relations are valid: $F_{m}^{\prime}=\frac{F_{m}}{k_{\sigma}} ; R_{m}^{\prime}=\frac{R_{m}}{k_{\sigma}}$, and the transformed scheme takes the form, as in Figure 2,b.

This transformation made it possible to eliminate the scattering conductivity when the preparation of the equivalent magnetic circuit, replacing the parameters of the permanent magnet by the reduced ones. Let us note, that

$$
F_{m}=h_{m} H_{C} ; R_{m}=\frac{h_{m} H_{C}}{S_{m} B_{r}}=\frac{h_{m}}{S_{m} \mu_{r} \mu_{0}},
$$

where $h_{m}$ - the length of the magnet in the direction of magnetization; $S_{m^{-}}$the area of magnet neutral section; $B_{r}$ - the residual induction of the magnet, $H_{c^{-}}$coercive force; $\mu_{r}$-relative permeability of a permanent magnet material.

Taking into account the relationship between the magnetic resistance and magnetic conductivity coefficient of reduction can be calculated, and through the corresponding magnetic conductivities:

$$
k_{\sigma}=1+\frac{\lambda_{\sigma}}{\lambda_{m}} .
$$

For the magnetic circuit in Figure $2 b$, as a singlecircuit one, the magnetic flux $\mathrm{F}_{\delta}$ is found from the obvious relation:

$$
\Phi_{\delta}=\frac{F_{m}^{\prime}}{R_{\delta}+R_{m}^{\prime}} .
$$

Taking into account the distribution of the magnetic fluxes in the magnetic system (Fig.1) equivalent circuit has the form as in Figure 3.
Due to the symmetry of the coupling design and based on the first Kirchhoff's law for magnetic circuits, the relation is valid:

$$
\Phi_{1}=\frac{\Phi_{\delta}-\Phi_{3}}{2} .
$$

Let us compose the equations according to the second Kirchhoff's law for this scheme:

$$
\left\{\begin{array}{l}
\Phi_{\delta}\left(R_{m 1}^{\prime}+R_{m 2}^{\prime}+R_{\delta}\right)+\Phi_{3} R_{T}=F_{m 1}^{\prime}+F_{m 2}^{\prime} \\
\Phi_{1}\left(R_{1}+R_{2}\right)-2 \Phi_{3} R_{T}=0
\end{array} .\right.
$$

Solving the obtained equation relative to the flow $\mathrm{F}_{\delta}$, we obtain:

$$
\Phi_{\delta}=\frac{4\left(F_{m 1}^{\prime}+F_{m 2}^{\prime}\right)}{4\left(R_{m 1}^{\prime}+R_{m 2}^{\prime}+R_{\delta}\right)+\left(1-k_{T}\right)\left(R_{1}+R_{2}\right)},
$$

where $k_{T}=\frac{R_{1}+R_{2}}{R_{1}+R_{2}+4 R_{T}}$.

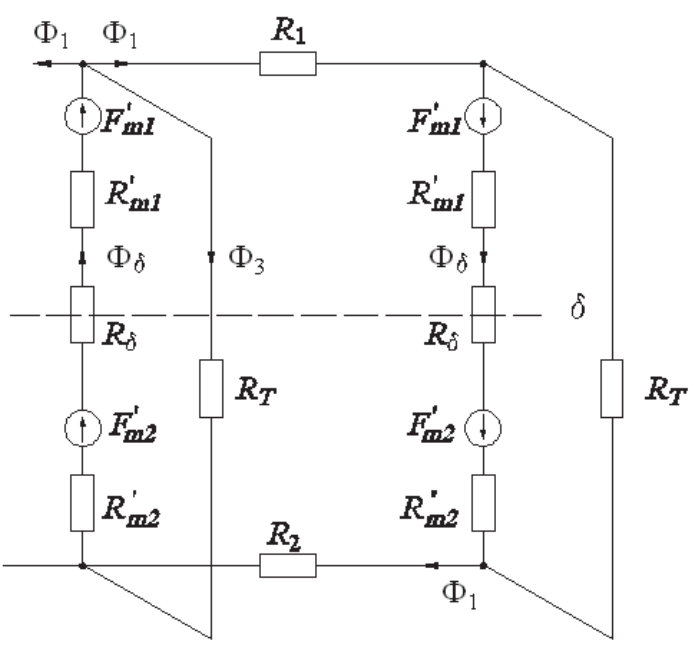

Fig. 3. Replacement scheme of the coupling magnetic circuit.

As follows from the expression, the magnetic resistances of the sections both in the transverse plane $\left(R_{1}, R_{2}\right)$ and in the longitudinal $\left(R_{T}\right)$ affect the magnitude of the magnetic flux in the air gap. Since their magnitude is determined by the geometry of the corresponding air gaps, there is some uncertainty in the estimation of the permanent magnets outer faces areas for each component of the magnetic flux. For engineering calculations the following algorithm is proposed to calculate the components of the useful flux based on the solution of two plane problems, based on the physical picture of the distribution of magnetic fluxes in the considered magnetic system:

1. The planar problem for the circuit in Figure 1,a is solved, and the magnetic flux in the air gap $F_{\delta 1}$ is calculated in neglect of the flows in the other plane. Thus, the magnetic circuit resistance of the magnetic system transverse contour is fixed. 
2. The plane problem for the circuit in Figure $1, \mathrm{~b}$ is solved, and the magnetic flux in the air gap $F_{\delta 2}$ is calculated in neglect of the flows in another plane. This fixes the level of resistance of the magnetic circuit in the longitudinal (axial) circuit.

3. From the assumption that the distribution of the areas in the cross section of the permanent magnets for the fluxes $F_{1}$ and $F_{3}$ (Fig.3) in the real system corresponds to the relationship between the flows calculated in item 1 and item 2 , the resistance of $R_{1}, R_{2}$ and $R_{T}$ is adjusted, since their value is inversely proportional to the corresponding areas.

4. The magnetic flux in the air gap is calculated according to the replacement scheme in Figure 3, taking into account the corrected value of the resistance as per item 3.

Let's consider each of the listed items of the calculation algorithm.

\subsection{Calculation of the magnetic flux Fo1}

The coefficient of reduction is calculated taking into account all the scattering fields along the perimeter of the permanent magnets so that the values of the magnets MMF can be used both for calculation of the fluxes of $F_{\delta 1}$ and $F_{\delta 2}$, and then of the flux of $F_{\delta}$. This takes into account the scattering conductivity $\lambda_{\sigma p}$ along the edges of the permanent magnets between the neighboring lateral faces of each magnet (the conductivity of the spherical quadrants, in Figure 1 a are not shown) [9]. Since the distance between the lateral faces of the magnets may be greater than half the $h_{m}$, in this case, in addition to the conductivity $\lambda_{\sigma 1}$ between the lateral faces of the neighboring magnets, the scattering conductivity $\lambda_{\sigma \sigma}$ along the lateral faces of each magnet should be added [9]. Then the conductivity of the magnet scattering of the upper half-coupling is defined as the sum $\lambda_{\sigma B}=\lambda_{\sigma 1}+\lambda_{\sigma \sigma}+\lambda_{\sigma p}$. The scattering conductivity between the side faces can be found from the formula [10]:

$$
\lambda_{\sigma}=\frac{k \Phi_{\sigma}}{2 h_{m} H_{c}}=0,5 k \mu_{0} \frac{l h_{m}}{\alpha_{c p}}
$$

where $k=1,05-1,1$ - coefficient that takes into account the bulging of the force lines along the path of closure of the scattering flux; $\alpha_{c p}=\left(\Delta+\Delta_{1}\right) / 2$.

The conductivities $\lambda_{\sigma \sigma}$ и $\lambda_{\sigma p}$ are found by the formulas [9]:

$$
\lambda_{\sigma \sigma}=2 \cdot 0,26 \mu_{0}\left(l+b_{1}\right) ; \lambda_{\sigma p}=4 \cdot 0,77 \mu_{0} h_{m} .
$$

Magnetic conductivity of the upper magnet:

$$
\lambda_{m 1}=\frac{S_{m 1} B_{r}}{h_{m} H_{c}} .
$$

Reduction coefficient is $k_{\sigma 1}=1+\frac{\lambda_{\sigma B}}{\lambda_{m 1}}$. The reduced MMF of the upper magnet is $F_{m 1}^{\prime}=\frac{h_{m} H_{c}}{k_{\sigma 1}}$. The reduced magnetic resistance of the upper magnet is:

$$
R_{m 1}^{\prime}=\frac{h_{m}}{S_{m 1} \mu_{r} \mu_{0} k_{\sigma 1}}
$$

Similarly, the reduced values of the MMF $F_{m 2}^{\prime}$ and the resistance $R_{m 2}^{\prime}$ of the lower magnet are calculated.

Since the permanent magnets are parallelepipeds, and the air gap is formed by the parallel faces (when the coupling is idling), the magnetic resistance of the air gap we find by the formula:

$$
R_{\delta}=\frac{\delta}{\mu_{0} l b_{c p}}
$$

where $b_{c p}=\left(b_{1}+b_{2}\right) / 2$.

When calculating the magnetic resistances $R_{1}$ and $R_{2}$, calculated expressions are used for the conductivity of air gaps between rectangular surfaces lying in one plane [9], corrected by an angle $\beta$ corresponding to the arrangement of the magnets along the circumference.

$$
\begin{aligned}
& R_{1}=\frac{1}{\frac{\mu_{0}}{\beta_{1}} l \ln \left(1+2 \frac{0,5 b_{1}+\sqrt{0,25 b_{1}^{2}+0,5 b_{1} \Delta_{1}}}{\Delta_{1}}\right)} \\
& R_{2}=\frac{1}{\frac{\mu_{0}}{\beta_{2}} l \ln \left(1+2 \frac{0,5 b_{2}+\sqrt{0,25 b_{2}^{2}+0,5 b_{2} \Delta_{2}^{\prime}}}{\Delta_{2}^{\prime}}\right)} .
\end{aligned}
$$

If the holders with permanent magnets are made of electrical steel, then for an approximate calculation, the resistance of the sections with steel can be neglected in comparison with the air gap resistance. Then we can take $R_{1}=R_{2}=0$, which will greatly simplify the calculations. After the decision has been made about the dimensions of the device magnetic circuit, a verification calculation is performed to determine the torque characteristics, taking into account the possible saturation of the sections with steel.

Thus, the magnetic flux $F_{\delta 1}$ in the air gap is equal to:

$$
\Phi_{\delta 1}=\frac{4\left(F_{m 1}^{\prime}+F_{m 2}^{\prime}\right)}{4\left(R_{\delta}+R_{m 1}^{\prime}+R_{m 2}^{\prime}\right)+R_{1}+R_{2}} .
$$

\subsection{Calculation of the magnetic flux For}

To calculate the magnetic flux $F_{\delta 2}$, in addition, we must find the resistance $R_{T}$. Its value depends on the ratio of the linear dimensions of the permanent magnets and the 
air gap size and is inverse of the corresponding conductivity $\lambda_{T}=g 2 b \mu_{0}$, where $g=f(m, n)$ is the geometric dimension function given graphically in [9]. In this case with $m=\left(2 h_{m}+\delta\right) / 2 h_{m}, n=b_{c p} / \delta$ the magnetic flux is:

$$
\Phi_{\delta 2}=\frac{F_{m 1}^{\prime}+F_{m 2}^{\prime}}{R_{m 1}^{\prime}+R_{m 2}^{\prime}+R_{\delta}+R_{T}} .
$$

\subsection{Correction of the resistance of the replacement circuit}

Below the expressions are presented for the corrected values of the magnetic circuit resistance:

$$
\begin{aligned}
& R_{1 k}=R_{1} \frac{\Phi_{\delta 1}+\Phi_{\delta 2}}{\Phi_{\delta 1}} ; \quad R_{2 k}=R_{2} \frac{\Phi_{\delta 1}+\Phi_{\delta 2}}{\Phi_{\delta 1}} \\
& R_{T k}=R_{T} \frac{\Phi_{\delta 1}+\Phi_{\delta 2}}{\Phi_{\delta 2}} .
\end{aligned}
$$

\subsection{Calculation of the magnetic flux Fo}

We substitute the expressions for the corrected resistances (17) into expression (8) and obtain the value of the magnetic flux:

$$
\Phi_{\delta}=\frac{4\left(F_{m 1}^{\prime}+F_{m 2}^{\prime}\right)}{4\left(R_{m 1}^{\prime}+R_{m 2}^{\prime}+R_{\delta}\right)+\left(1-k_{T}\right)\left(R_{1 k}+R_{2 k}\right)},
$$

where $k_{T}=\frac{R_{1}+R_{2}}{R_{1 k}+R_{2 k}+4 R_{T k}}$.

Then this value of the magnetic flux is substituted into expression (2) for calculating the torque of magnetic coupling.

\section{Modeling by the finite element method}

The above procedure was verified with the help of the finite element method on $2 D$-models of the magnetic coupling. The difference between the values of the failure torque, calculated by the proposed method, and the torque values, obtained by the finite element method, for specific specified sizes of magnetic couplings does not exceed $10 \%$. It should be noted that the accuracy of the calculation is largely determined by the thoroughness in determining the geometry of the magnetic flux components paths and the correct application of the calculated formulas for the conductivity of the corresponding magnetic gaps [9].

Figure 4 shows the magnetic field pattern for $2 D$ analysis at the rotation angle $\mathrm{Q}=0^{\circ}$. Figure 5 shows the pattern of the magnetic field in the axial direction. Since the calculation of the $2 D$ model by the finite element method gives us the values of the magnetic fluxes $F_{\delta 1}$ and $F_{\delta 2}$, we can control and, if necessary, adjust the value of the scattering conductivities, involved in determining the reduction coefficient, and the corresponding MMF of the permanent magnets.

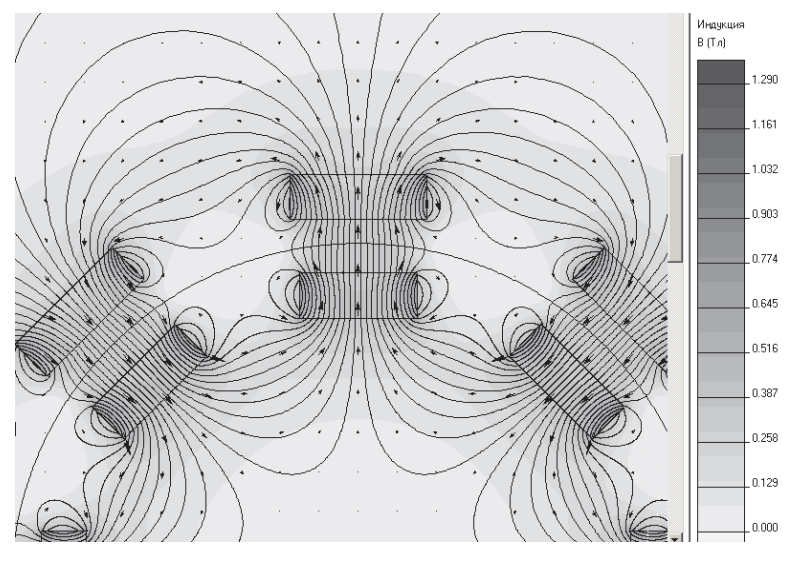

Fig. 4. Magnetic field of a plane problem for the rotation angle $\mathrm{Q}=0^{\circ}$.

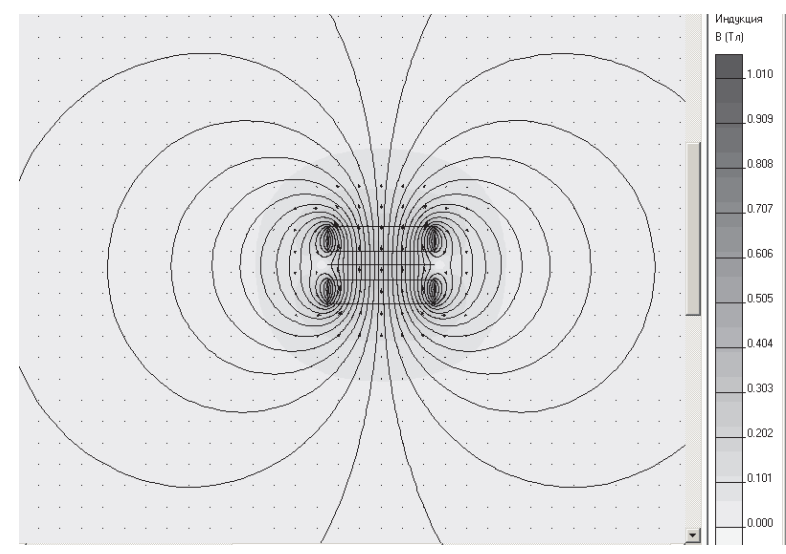

Fig. 5. Magnetic field in the axial direction.

\section{Conclusion}

The proposed method makes it possible to calculate the failure torque of magnetic couplings, in which the reinforcement and structural parts are made of nonmagnetic materials $(\mu=1)$ and can be recommended for verification calculation of magnetic couplings at the stage of elaborating design options and choosing the number of poles pairs.

\section{References}

1. A. Ja. Krasil'nikov, Vestnik mashinostroenija [Bulletin of Machine Building], 4 (2017) (in Russian)

2. A. Ja., Krasil'nikov, A.A. Krasil'nikov, Vestnik mashinostroenija [Bulletin of Machine Building], 6 (2009) (in Russian)

3. L.Thierry, S.Mezani, Simple Analytical Expressions for the Force and Torque of Axial Magnetic Couplings. IEEE Transactions on Energy Conversion, (2012)

4. M. H. Nagrial, J. Rizk, A. Hellany, World Academy of Science, Engineering \& Technology International Journal of Mechanical, Aerospace, Industrial, Mechatronic and Manufacturing Engineering, 5(7) (2011) 
5. D. MeeKer, Finite Element Method Magnetics. Available at: http://femm.berlios.de/ (Accessed 20 February 2017)

6. J. Fontchastagner, Y.Lefèvre, F.Messine, IEEE Transactions on Magnetics, 45(3) (2009)

7. S. Mohammadi, M. Mirsalim, S. Vaez-Zadeh. IEEE Transactions on Energy Conversion, 29, 1 (2014)

8. Je.V Lapshin, I.V. Tolstouhov, Trudy Mezhdunarodnogo Simpoziuma "Nadezhnost' $i$ kachestvo" [Proc.Int.Symp."Reliability and quality”], Penza, 2, (2014) (in Russian).

9. Pjatlin Ju.M., Postojannye magnity. Spravochnik. [Permanent magnets. Directory], (Moscow, Energy, 1971), (in Russian)

10. V.D. Kosulin, Scientific and technical conference "Zavalishenskie readings, St. Petersburg, SUAI, (2011) (in Russian) 